\section{Cardiovascular effects of reflexology in healthy individuals: evidence for a specific increase in blood pressure}

\author{
Elisabeth Ruiz-Padial, \\ Nieves Torres López, \\ Javier Luna Bujaldón, \\ Isabel Espadas Villanueva, \\ Gustavo A. Reyes del Paso \\ University of Jaén, Jaén, Spain.
}

\section{Abstract}

The present study evaluates the cardiovascular effects of reflexology in a healthy sample. Forty-one participants were randomly assigned to one of three experimental groups: reflexology $(n=15)$, non-professional foot massage $(\mathrm{n}=14)$, and a waiting time control group $(\mathrm{n}=12)$. Dependent variables were systolic, diastolic and mean blood pressure, inter-beat interval, heart rate variability and baroreceptor reflex sensitivity measured pre- and post- interventions. The study was performed during three 40 -min sessions separated by weekly intervals. Results show that the three manipulations produce similar increases in inter-beat interval, heart rate variability and baroreceptor reflex sensitivity. Reflexology specifically produces an increase in blood pressure, which increases gradually over the three sessions. The parallel increase in heart rate variability and baroreceptor reflex sensitivity together with the increase in blood pressure suggest that reflexology is associated with a co-activation of both sympathetic and parasympathetic branches of the Autonomic Nervous System. These changes could be helpful in optimizing homeostatic activity, promoting the healing process and increasing the human organism's capacity to respond adaptively to internal and external challenges. Finally, the observed physiological changes in the waiting-time control group shows the relevance of habituation processes and suggests the need for addition of waiting-time control groups in future studies.

\section{Introduction}

Reflexology is a form of healing already practiced more than 4000 years ago in Ancient China and Egypt. ${ }^{1}$ Its basic premise is that all glands, organs and systems of the body are represented on the feet, in such a way that by pressing reflex points on the feet it is possible to produce specific effects in those glands, organs or systems. It is therefore assumed that reflexology helps to maintain overall health and relieve symptoms of stress and disease. ${ }^{1}$

This technique has been used for centuries and reflexologists claim its effectiveness in alleviating a wide range of diseases and afflictions, such as pre-menstrual symptoms, ${ }^{2}$ lower back pain, ${ }^{3}$ diabetes ${ }^{4}$ cancer ${ }^{5}$ multiple sclerosis, ${ }^{6}$ anxiety, ${ }^{7}$ asthma, ${ }^{8}$ etc. However, the methodological quality of most randomized controlled studies is poor and the few well-controlled studies show inconclusive results. ${ }^{9}$

There is recent evidence from a neuroimaging study supporting the basic assumption underlying foot reflexology. Nakamaru and colleagues 10 found that reflexological stimulation of foot reflex areas activated the somatosensory cortical areas of the corresponding body parts. This finding is relevant since it demonstrates that at least some parts of the body may have reflex points in the foot. However, some important aspects remain unclear. Does the reflexology affect the functioning of the organs that are stimulated? How exactly does reflexology affect the healing process? It has been proposed that reflexology stimulates the circulatory and lymphatic systems, parasympathetic activity, restorative sleep, etc,. while other more secondary mechanisms such as relaxation, placebo effect, the therapeutic interaction or the well known benefits of skin-to-skin contact have been also considered.1,11,13

The skin-to-skin contact factor could be an important mediating mechanism in the therapeutic effects of massage therapies in general. Interpersonal touch has been shown to be related to health and numerous studies have related physical contact to decreases in blood pressure and heart rate.14,15 Skin contact is one of the primary mechanisms for the release of oxytocin, a neuropeptide hormone with an important protective effect on the autonomic and cardiovascular systems. Oxytocin secretion inhibits alpha-adrenergic and hypothalamic pituitary-adrenal activity, reduces catecholamine, blood pressure and cardiac output levels, and enhances vagal activity. ${ }^{14,16-19}$ It is essential to distinguish between the simple effects of skin-to-skin contact and the specific effects of reflexology in order to be able to confirm the efficacy of reflexology.

For two main reasons it is particularly important to focus on the effect that reflexology may have on cardiovascular variables. On the one hand, one of the major explanations for the underlying mechanisms of reflexology is its effect on the circulatory system, ${ }^{12}$ so it could be expected to find changes in cardiovascular parameters after reflexology. On the other hand, some cardiovascular measures, such as heart rate variability and baroreceptor reflex sensitivity, have been demonstrated to be good indices of the organism's auto-regulatory capacity and health. ${ }^{20-25}$
Correspondence: Elisabeth Ruiz-Padial, Universidad de Jaén, Campus Las Lagunillas, Edif. C-5, 23071 Jaén, Spain.

Tel. +34.953.213.451 - Fax: +34.953.211.881.

E-mail: erpadial@ujaen.es

Key words: reflexology, foot massage, inter-beat interval, heart rate variability, baroreceptor reflex sensitivity.

Acknowledgments: this research was supported by a grant from the Junta de Andalucía (Research Group: HUM338).

Contributions: ERP, design and analysis of the physiological data, review of the literature, writing of the manuscript; NTL, conception of the study, interpretation of the results; JLB, conception, design and drafting of the paper, processing of the self-report measures and the statistical analysis. IEV, conception and design of the study, drafting and the review of the article; GARPaso, design of the study, analysis and interpretation of the physiological results, writing of the paper

Conflict of interest: the authors report no conflicts of interest.

Received for publication: 23 September 2011. Revision received: 30 December 2011.

Accepted for publication: 19 January 2012.

This work is licensed under a Creative Commons Attribution NonCommercial 3.0 License (CC BYNC 3.0).

(C) Copyright E. Ruiz-Padial et al., 2012

Licensee PAGEPress, Italy

Alternative Medicine Studies 2012; 2:e4

doi:10.4081/ams.2012.e4

Non-invasive evaluation of autonomic integrity and functioning can be performed by means of heart rate variability, a measure of regular fluctuations in heart rate over time. ${ }^{26}$ The baroreceptor reflex constitutes another important source of autonomic regulation. The arterial baroreceptors are stretch receptors located in the wall of the carotid sinus and the aortic arch and as such are a basic mechanism for the regulation of blood pressure. Increases in blood pressure stretch the walls, stimulating the baroreceptors, which in turn set off a reaction through the brainstem centers producing a reduction in heart rate, the contractility of the heart, and vascular tone, leading in turn to a reduction in blood pressure. The opposite occurs when blood pressure decreases. In this way, the activity of the baroreflex prevents rapid swings in blood pressure by eliciting compensatory adjustments in both parasympathetic and sympathetic activation. ${ }^{27}$ The cardiac branch of the reflex that relates blood pressure to inter-beat interval is a powerful source of vagal afferent input to the central nervous system. Moreover it 
is one of the most important physiological mechanisms affecting efferent cardiac vagal activity and the main generator of autonomic parasympathetic measures such as respiratory sinus arrhythmia and heart rate variability. ${ }^{27-29}$ It is well established that autonomic parasympathetic control is strongly associated with cardiovascular diseases and is a powerful prognostic indicator of cardiovascular health. 23,24

The arterial baroreceptors, furthermore, constitute the starting point of an afferent pathway by which cardiovascular function modulates central nervous activity. Responding to mechanical stretch of the carotid and aortic vessel walls, the baroreceptors trigger a global inhibitory effect on brain activity that decreases somatic muscle tone, stimulates sleep, inhibits spinal somatic sensory pathways, reduces anger, anxiety and pain and attenuates behavioural reactions to painful and aversive stimuli. ${ }^{30-32}$ The baroreflex's inhibitory modulation of cerebral structures related to emotion and arousal is the foundation for techniques for strengthening the baroreceptors such as those that have traditionally been used in Russia for the treatment of psychosomatic disorders such as asthma, arterial hypertension, insomnia, etc. ${ }^{33,34}$ For all these reasons, heart rate variability and baroreceptor functioning are good candidates for the evaluation of the efficacy of reflexology.

To the best of our knowledge, few studies have explored the effects of reflexology on cardiac variables and most of them present important methodological weakness. Frankel 35 found that reflexology and foot massage reduced baroreceptor reflex sensitivity. However, this result must be considered cautiously. In addition to using a small sample, the technique used (the valsalva manoeuvre) is a now obsolete invasive method. ${ }^{36}$ Furthermore, some contradictory associations found in this study such as the negative relation between baroreceptor reflex sensitivity and respiratory sinus arrhythmia are contrary to current evidence, 28,37 therefore suggesting the need for new studies evaluating the effects of reflexology on baroreceptor functioning using more upto-date methodology.

Some studies have evaluated the effects of reflexology on heart rate variability. Joseph et $a l .38$ and Zhen et al. ${ }^{39}$ found decreases in heart rate variability during reflexology in comparison to a relaxed sitting position. This result seems contra-intuitive and is contrary to findings from other types of massage techniques such as the myofascial trigger-point massage, which has been shown to increase heart rate variability. 40

Other studies have found a reduction in systolic blood pressure and heart rate following reflexology 7 and other methods such as deeptissue massage therapy. ${ }^{41}$ Furthermore, Ejindu $^{42}$ found that while facial massage produces a reliable decrease in blood pressure, the effects of foot massage on blood pressure are negligible. Studies using electrical massage devices, which perform pulse-synchronized massage with air cuffs, have found that massage pulses increase peripheral vascular blood flow, an effect that can influence venous return and subsequent autonomic nerve activity. 43

In addition to using small samples, many of these studies do not include appropriate massage control groups, making it difficult to conclude whether the effects observed are due to the reflexology or to the skin-to-skin contact. More importantly, the studies aiming to evaluate the effects of reflexology on physiological parameters usually utilize a pre- post-intervention assessment. None of these studies has controlled for the effect of habituation to the setting in which the intervention is performed. Habituation is a non-associative learning process that implicates a decrease in physiological indices as the subject gets used to the situation. The environmental context used in these intervention studies is new for the subject (devices, wires, interpersonal contact, etc.) and therefore has arousing properties. Thus any decrease in physiological indices might also be due to this habituation process.

The aim of the present study was to evaluate the cardiovascular effects of reflexology in comparison with a foot-massage procedure (to control for the skin-to-skin contact factor) and a waiting-time control group (to control for the habituation process) in healthy participants. Dependent variables were blood pressure, inter-beat interval, heart rate variability and baroreceptor reflex sensitivity. Furthermore, the effects of the three procedures on selfreported anxiety were assessed.

\section{Materials and Methods}

\section{Subjects}

Forty-one psychology students (20 male and 21 female) from the University of Jaén voluntarily participated in the study. Their mean age was $20.85 \pm 2.96$ years and all of them were healthy (none presented a diagnosis, history or symptoms of any disease). Our students were informed in classroom that they could sign in a list if they were interested in participating in a study on the effects of different techniques of alternative medicine on psychophysiological responses. Participants were randomly assigned to one of three experimental groups: 15 to the reflexology group (R), 14 to the nonprofessional foot massage (M) and 12 to the waiting time control group (C). They did not know the specific goals of the study nor which group they were assigned to. The study protocol was approved by the Bioethics Committee of the University of Jaén and all the participants signed the informed consent form.

\section{Apparatus}

Blood pressure was recorded continuously and non-invasively on a beat-to-beat basis with the Ohmeda 2300 BP monitor (Ohmeda, Louisville, KY) from the middle phalanx of the third finger of the right hand. The hand was positioned at the level of the heart. This device measures blood pressure following the vascular wall unloading principle and has been validated against intra-arterial continuous recording. ${ }^{4}$ Electrocardiogram (ECG) was obtained from Einthoven Lead II using a Biopac ECG amplifier. Data acquisition and recording of both ECG and blood pressure were carried out at $500 \mathrm{~Hz}$ using a Biopac MP100 system (Biopac System Inc., Goleta, CA).

\section{Experimental group manipulations}

For the reflexology group, the Ingham method of reflexology was used, carried out by one of the authors, an expert reflexologist. Each session lasted $40 \mathrm{~min}$ (20 min each foot) and started with the warming of one foot followed by pressing the reflex point called solar plexus seven times according to the respiration of the participant, after which the reflexological massage covered urinary, circulatory, immune, endocrine and central nervous systems as well as the spinal column, ending with relaxation, after which the same sequence was repeated for the other foot. Since skin-to-skin contact may be one of the mediation mechanisms in the efficacy of reflexology, another group of participants received a non-professional foot massage lasting $20 \mathrm{~min}$ for each foot. This massage was carried out by a student who had no previous experience as a masseur or reflexologist. To discard the effects of habituation to the laboratory environment on the dependent variables, a waiting time control group was used. They received no treatment and were seated for $40 \mathrm{~min}$ in a darkened room under the same conditions as the other two groups.

\section{Self-reported variables}

Anxiety was assessed with the Spanish adaptation of the State-Trait Anxiety Inventory (STAI). ${ }^{45}$ We also developed two scales to evaluate the pleasantness and pain associated with the two massage procedures. Participants were asked to rate the pleasantness and painfulness of both massage procedures using a 0 to 10 scale ( 0 meaning not at all and 10 extremely pleasant and painful, respectively).

\section{Procedure}

Each participant attended three sessions separated by weekly intervals. All the sessions were conducted in the Psychophysiology laboratory, in a temperature-controlled room (mean temperature was $23.6^{\circ} \mathrm{C}$ ). Prior to the first session, an interview was conducted in order to evaluate 
health status and pharmacological or psychiatric treatment. After receiving instructions, the participant signed an informed consent form, as we described above, and answered some questions related to age, exercise, menstrual cycle (women only), any type of medications, alcohol and food or drink consumed before the session. After this, each participant completed the two scales of the STAI (A/R -trait- and A/E -state). Then electrodes were placed on the participant and he/she was asked to seat comfortably in a reclined position. After a 10 -min rest period, a 5-min resting baseline of BP and ECG recording was made after which the participant either received a 20-min massage on each foot (groups R and M) or was left alone for 40 minutes (group C). Immediately afterwards, a further 5-min recording was taken. Finally, all participants were given the State form of the STAI and the $\mathrm{R}$ and $\mathrm{M}$ groups also completed the scales for pleasantness and painfulness associated with the massage. With the exception of the initial interview, the instructions and signing of the informed consent form, the second and third sessions were identical to the first one. Participants were instructed to refrain from drinking caffeine or alcohol or performing vigorous exercise for $2 \mathrm{~h}$ prior to the sessions.

\section{Data reduction and analysis}

Blood pressure was analyzed by obtaining the systolic, diastolic and mean values for each beat. Inter-beat interval was obtained from the interval between successive ECG R-R waves. Baroreceptor function was evaluated with a time-domain program ${ }^{46}$ which locates either i) sequences of three to six consecutive cardiac cycles in which systolic blood pressure increases (of at least $1 \mathrm{mmHg}$ to each beat) in combination with an increase in inter-beat interval (of at least $2 \mathrm{~ms}$ in each beat) or ii) sequences in which the decrease of systolic blood pressure is accompanied by a decrease in inter-beat interval (following the same criteria of minimum change). Each systolic value is paired with the inter-beat interval calculated from the very next heart beat -a lag of one beat- which is associated with better estimates of baroreceptor reflex sensitivity. 47 When one of these sequences is detected, the corresponding regression line is computed across all pulses in that particular sequence. Baroreceptor reflex sensitivity is expressed as the change in interbeat interval (in ms) per mmHg change in systolic blood pressure and is measured by the slope of the regression line. Heart rate variability was measured in the time-domain with the root mean square successive difference (RMSSD) of the inter-beat interval time-series. There is a strong association between RMSSD and respiratory sinus arrhythmia and parasympathetic outflow. 48,49 Finally, as an index of blood pressure variability, the RMSSD of systolic blood pressure was also obtained.
Homogeneity of variances and normality assumptions were assessed with the Levene and Kolmogorov-Smirnov tests, respectively. All variables analyzed fulfills the requirements of parametric statistical analysis. We detect baseline group differences for inter-beat interval $(\mathrm{F}(2,40)=6.38, P=0.004)$ and mean blood pressure $(\mathrm{F}(2,40)=3.643, P=0.036)$, as well as trends for systolic blood pressure $(\mathrm{F}(2$, $40)=1.94, P=0.157)$ and diastolic blood pressure $(\mathrm{F}(2,40)=2.38, P=0.106)$. In order to control such group differences, statistical analyses for the above parameters were performed with a $3(\times 3 \times 2)$ repeated measures ANCOVA the first factor between-group (Condition, manipulated at three levels: reflexology, foot massage and control), and two repeated-measures factors: session, with three levels (session 1, 2 and 3 ) and time, with two levels (pre- and postintervention or beginning and end of the session for the waiting time control group).The baseline value during the first session was introduced as covariable. The remaining parameters (systolic blood pressure variability, inter-beat interval variability, baroreceptor reflex sensitivity and the self-reported measures) were analyzed with a $3(\times 3 \times 2)$ repeated measures MANOVA, Effect sizes were calculated with the $\eta^{2}$ parameter. Post-hoc comparisons were performed with the Bonferroni procedure to control for the number of comparisons performed.

\section{Results}

\section{Physiological measures}

\section{Blood pressure}

Average values of systolic, diastolic and mean blood pressure through the course of the

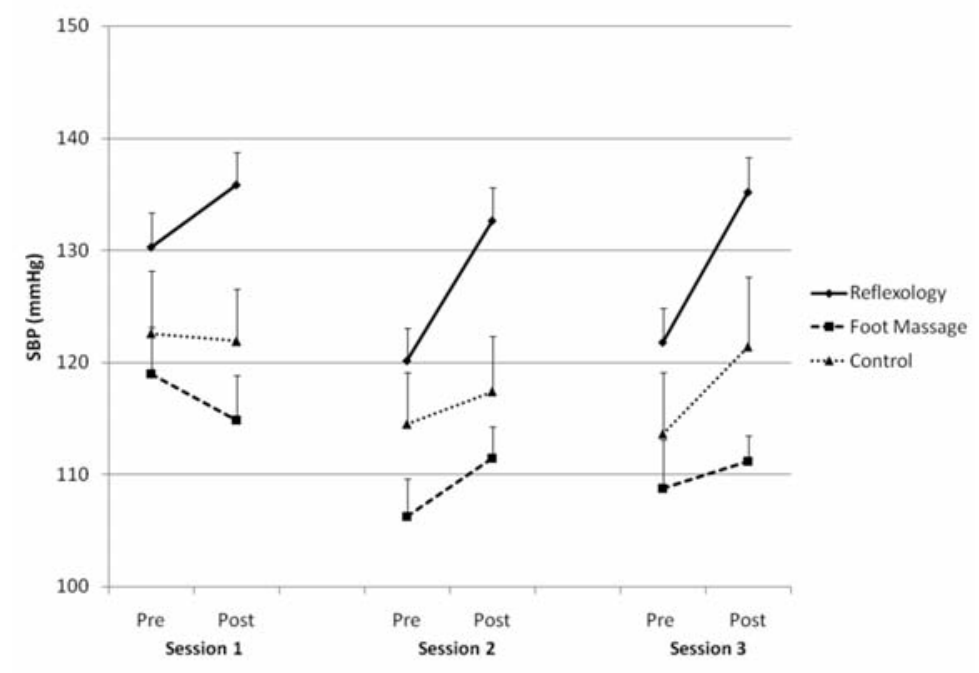

Figure 1. Systolic blood pressure during the three sessions as a function of conditions. Bars indicate standard errors of the mean. study are displayed in Figures 1 - 3. For systolic blood pressure the ANCOVA shows significant effects in the time $(F(1,37)=11.91, P=0.001$, $\left.\eta^{2}=0.243\right)$, condition $(F(2,37)=7.85, P=0.001$. $\left.\eta^{2}=0.298\right)$ and sessions $(F(2,36)=4.59$, $\left.P=0.017, \eta^{2}=0.203\right)$ factors. The interactions time $\mathrm{x}$ condition $(F(2,37)=10.29, P<0.0001$, $\left.\eta^{2}=0.3585\right)$ and time $\times$ sessions $(F(2,36)=4.55$, $\left.P=0.017, \eta^{2}=0.201\right)$ were also significant.

The analysis of the interaction TimexCondition shows that in the reflexology group the Time factor was significant $\left(F(1,14)=33.90, P<0.0001, \eta^{2}=0.708\right)$. As can be observed in Figure 1, the reflexology intervention increases blood pressure in that group. In the foot massage group, the Time factor is significant as a function of Sessions $\left(F(2,12)=9.11, P<0.01, \eta^{2}=0.602\right)$. In this group the Pre-Post comparison was significant only in the second session $(F(1,13)=5.20$, $\left.P<0.05, \quad \eta^{2}=0.284\right)$ where foot massage increases blood pressure. In the control group the Time factor was not significant. As shown by the significant main Condition factor effect, the increase in blood pressure after reflexology results in greater levels of blood pressure in the reflexology group than in the foot massage group $(P<0.0001)$ and the control group $(P<0.05)$, while the latter two groups did not differ from one another $(P=0.292)$. Table 1 displays the complete post-hoc analysis for systolic blood pressure. As can be observed, the reflexology group shows higher blood pressure values than the other two groups in the Post periods of the three sessions, while the foot massage and control groups did not show any difference.

The effect of the Session factor is explained by a decrease in blood pressure in the course of the sessions that is especially larger in the baseline periods and in the comparison between the first and the second sessions 
$(t=4.84, \mathrm{P}<0.0001)$. The interaction Timex Sessions is explained by the Time factor, which was significant in the second $(F(1,38)=27.26$, $\left.\mathrm{P}<0.0001, \eta^{2}=.418\right)$ and third $(F(1,38)=15.04$, $\left.P<0.0001, \eta^{2}=0.284\right)$ sessions, but not in the first one.

The results of the ANCOVA for diastolic and mean blood pressure are displayed in Table 2 . As can be observed, the effects are similar to those found for systolic blood pressure.

As regards systolic blood pressure variability, the MANOVA show significant effects in the Time factor $\left(F(1,38)=8.89, P<0.005, \eta^{2}=0.19\right)$. As can be observed in Figure 4, systolic blood pressure variability increases after the intervention, especially for the reflexology and foot massage groups, but the interaction TimexCondition does not reach significance.

\section{Inter-beat interval}

The Time factor was significant $\left(F(1,37)=57.45, P<0.001, \eta^{2}=0.456\right)$ as was the interaction Time $\times$ Sessions $(F(2,36)=4.23$, $\left.P<0.05, \eta^{2}=0.187\right)$. As can be observed in Figure 5, inter-beat interval increases in all three groups in the post-period. Although this effect is observed in the three sessions, its statistical significance is reduced as a function of sessions $\left(F(1,38)=63.56, P<0.0001, \eta^{2}=0.624\right.$; $F(1,38)=27.6, P<0.0001, \eta^{2}=0.421 ; F(1,38)=$ $14.5, P<0.0001, \eta^{2}=0.277$; respectively for sessions 1,2 , and 3 ).

As regards heart rate variability, the Time factor was also significant $(F(1,38)=30.21$, $\left.P<0.0001, \eta^{2}=0.443\right)$ as was the interaction Time $\times$ Sessions $\left(F(2,37)=3.326, P<0.05, \eta^{2}=\right.$ 0.152 ). As can be observed in Figure 6, heart rate variability increases in the three groups after intervention and again this effect decreases as a function of sessions $(F(1,38)=27$, $P<0.0001, \eta^{2}=0.417 ; F(1,38)=11.00, P<0.01$, $\eta^{2}=0.225 ; \quad F(1,38)=9.14, P<0.01, \eta^{2}=0.194$; respectively for sessions 1,2 , and 3 ).

\section{Baroreceptor reflex sensitivity}

The only significant effect found was for the Time factor $\left(F(1,38)=19.27, P<0.0001, \eta^{2}=\right.$ 0.337 ). As can be observed in Figure 7, baroreceptor reflex sensitivity increases in the three groups after intervention.

\section{Self-report measures}

\section{Anxiety}

A main effect of Time $(F(1,38)=40.89$, $\left.P<0.0001, \eta^{2}=0.518\right)$ and a significant interaction Time $\times$ Condition $(F(2,38)=6.37, P<0.004$, $\eta^{2}=0.251$ ) were observed. As displayed in Figure 8, anxiety decreases after reflexology $\left(F(1,14)=19.27, P<0.001, \eta^{2}=0.579\right)$ and foot massage $\left(\mathrm{F}(1,13)=26.52, P<0.0001, \eta^{2}=0.671\right)$ but does not change in the waiting control group.

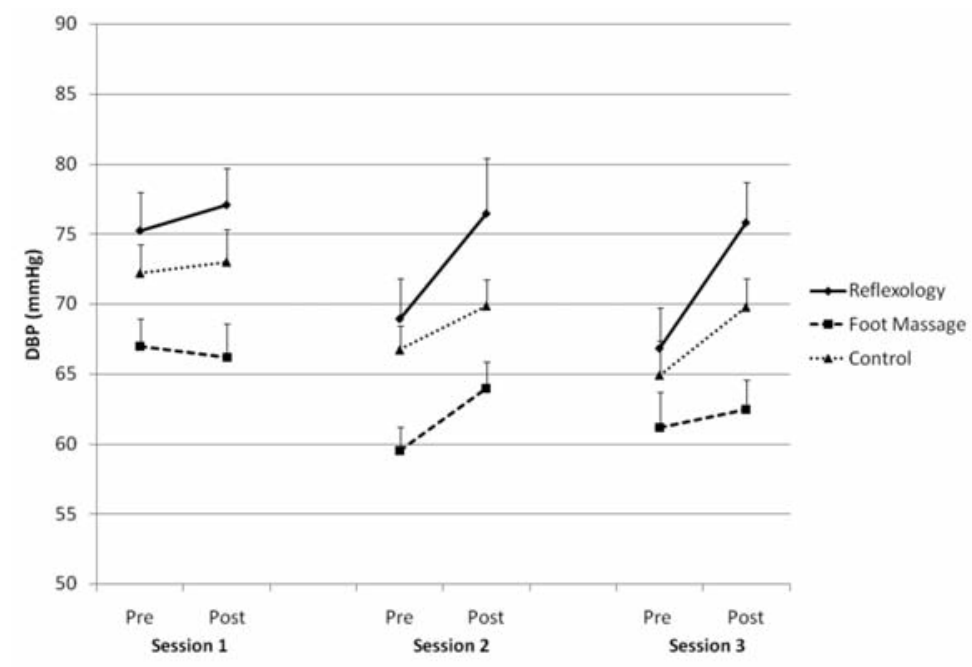

Figure 2. Diastolic blood pressure during the three sessions as a function of conditions. Bars indicate standard errors of the mean.

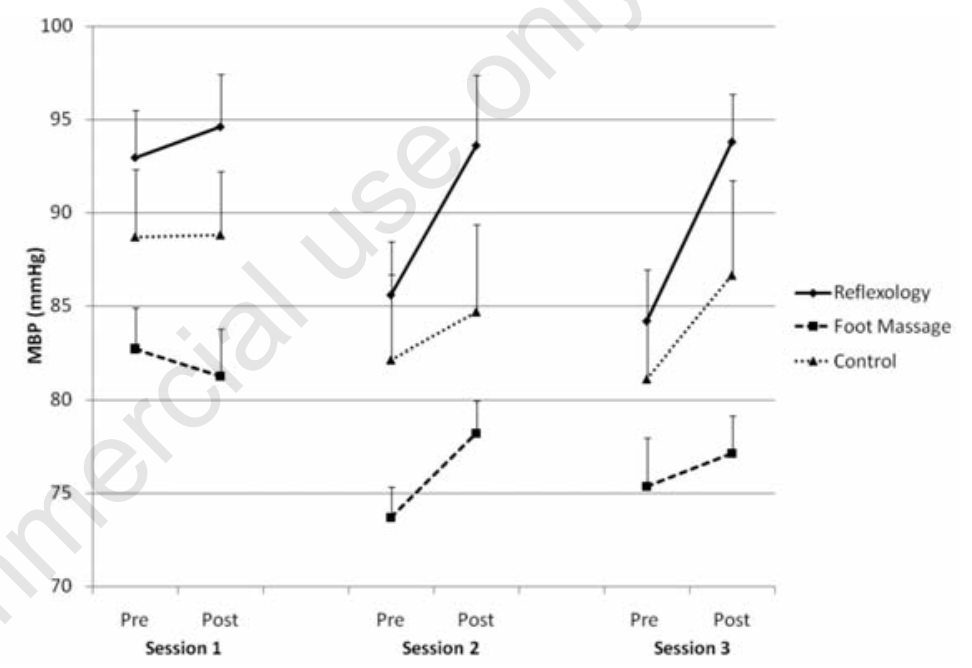

Figure 3. Mean blood pressure during the three sessions as a function of conditions. Bars indicate standard errors of the mean.

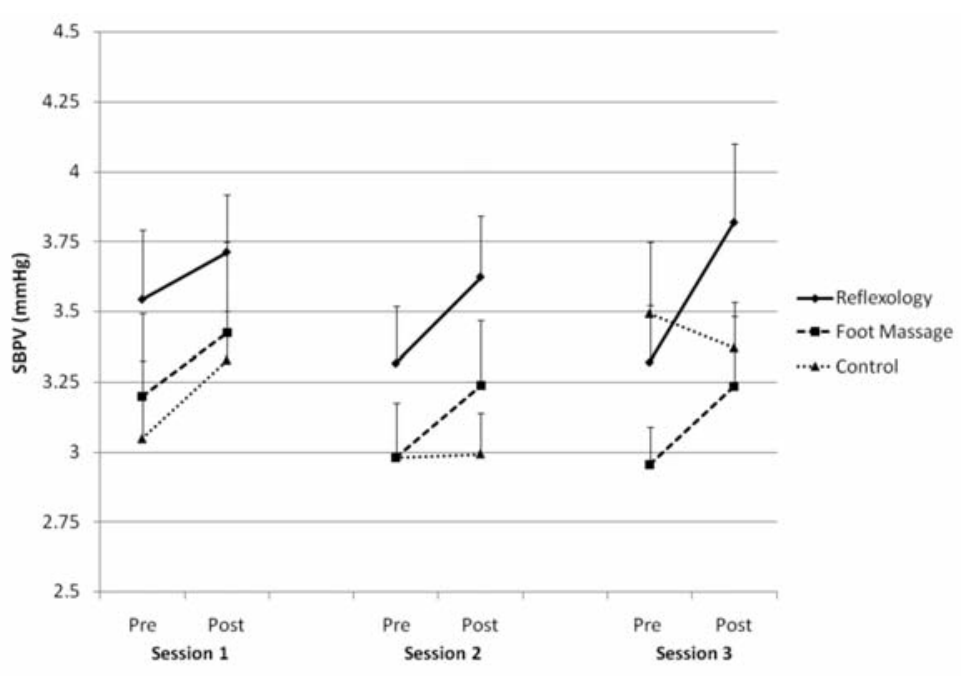

Figure 4. Systolic blood pressure variability during the three sessions as a function of conditions. Bars indicate standard errors of the mean. 


\section{Pleasantness and pain in the two massage procedures}

Both reflexology and non-professional foot massage were evaluated as equally pleasant $(8.46 \pm 1.2$ for the R group and $8.98 \pm 1.04$ for the $M$ group). None of the main factors or their interaction were significant. As regards intensity of the pain, results showed significant effects of Condition $(F(1,27)=20.49, P<0.0001$, $\left.\eta^{2}=0.431\right)$, Session $(F(2,26)=5.174, P<0.05$, $\left.\eta^{2}=0.285\right)$ and the interaction Condition $\times$ Session $\left(F(2,26)=4.04, P<0.05, \eta^{2}=0.237\right)$. As can be observed in Figure 9, the reflexology group rated the massage as more painful, especially during the first session $(F(2,13)$ $\left.=5.47, P<0.05, \eta^{2}=0.457\right)$.

\section{Discussion}

Our results indicate that reflexology produces an increase in blood pressure within session (systolic, diastolic and mean blood pressure) in comparison with the foot massage and the waiting-control group. No differences were found between the three conditions regarding the other physiological measures. Inter-beat interval, heart rate variability and baroreceptor reflex sensitivity increase after reflexology, foot massage and waiting time. However, the increase in inter-beat interval and heart rate variability after the three procedures decreases over the course of the sessions, showing a habituation process. It is noticeable that although inter-beat interval, heart rate variability and baroreceptor reflex sensitivity (indices of heart activity) all increase, blood pressure also increases. This suggests that the changes in blood pressure can be explained by changes in vascular factors after reflexology.

Anxiety levels decrease in both the reflexology and foot-massage groups after intervention in each session. However, no change was observed in the waiting control group. Both types of massage were rated equally as highly pleasant, although reflexological massage was evaluated as painful as well.

Both heart rate variability and baroreceptor reflex sensitivity are indices of parasympathetic cardiac control.22,28,37 Thus, the increased heart rate variability and baroreceptor reflex sensitivity during our three experimental manipulations point to an increase in cardiac vagal activity that is not specific to reflexology since it also occurred during the foot-massage and even the normal habituation process. So at a conservative level, we can speculate that most of this parasympathetic effect can be explained as due to habituation to the laboratory environment.

Our results concerning baroreceptor reflex sensitivity are contrary to those observed by
Table 1. Mean systolic blood pressure differences between conditions for each of the experimental periods. Post-hoc comparisons were performed with the Bonferroni procedure. $\mathrm{R}$, reflexology; $\mathrm{M}$, foot massage; $\mathrm{C}$, control group.

\begin{tabular}{lcccccc} 
& \multicolumn{2}{c}{ Session 1 } & \multicolumn{2}{c}{ Session 2} & \multicolumn{2}{c}{ Session 3 } \\
& Pre & Post & Pre & Post & Pre & Post \\
R versus M & 11.24 & $20.95^{* *}$ & $13.82^{*}$ & $21.21^{* *}$ & 12.97 & $23.98^{* *}$ \\
\hline R versus C & 7.68 & $13.93^{*}$ & 5.65 & $15.24^{* *}$ & 8.17 & $13.79^{*}$ \\
M versus C & -3.55 & -7.02 & 8.18 & -5.97 & -4.79 & 10.19 \\
\hline
\end{tabular}

$\mathrm{P}^{*}<0.05,{ }^{* *}<0.01$

Table 2. Results of the $3(\times 3 \times 2)$ repeated measures ANCOVA for diastolic (DBP) and mean blood pressure (MBP). The baseline value during the first session was introduced as covariable. Simple main effects $(\mathrm{T}=$ Time, $\mathrm{S}=\mathrm{Sessions}$ and $\mathrm{C}=\mathrm{Condition})$ and significant interactions are displayed.

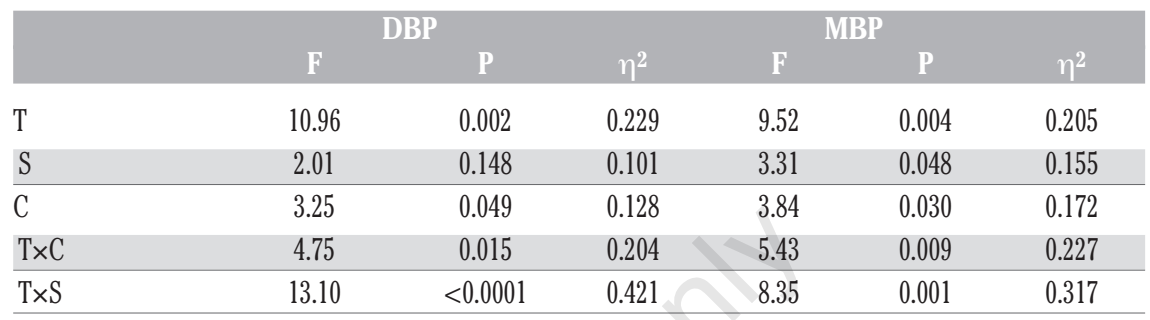

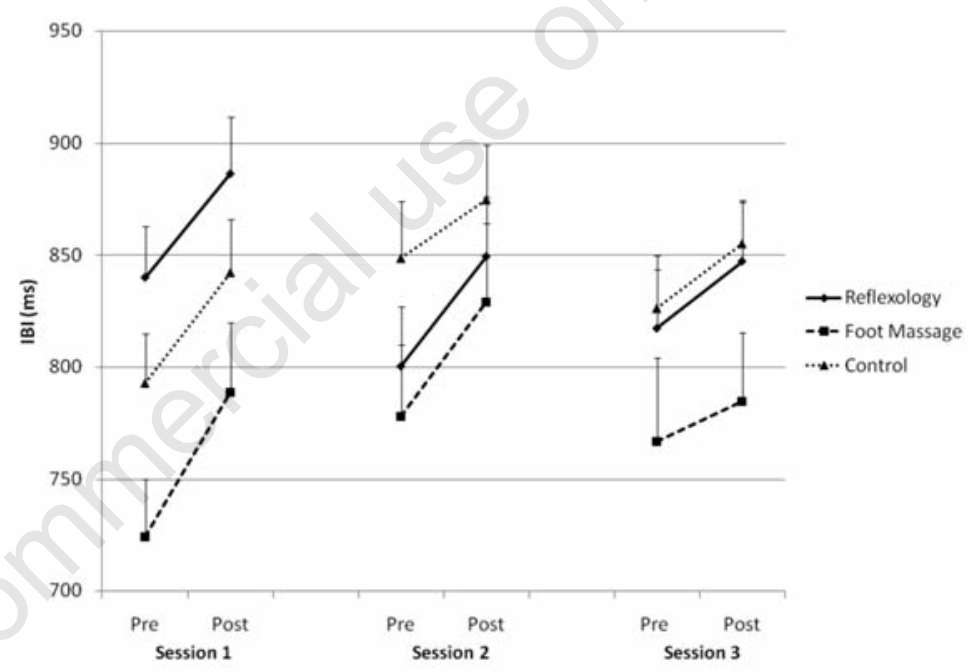

Figure 5. Inter-beat interval during the three sessions as a function of conditions. Bars indicate standard errors of the mean.

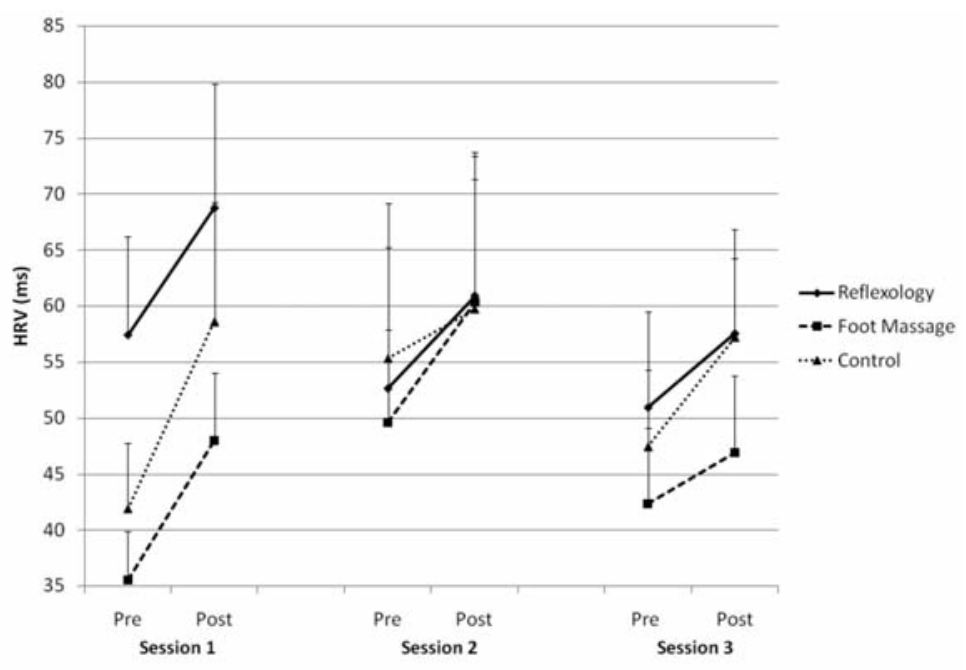

Figure 6. Heart rate variability (RMSSD) during the three sessions as a function of conditions. Bars indicate standard errors of the mean. 
Frankel. ${ }^{35}$ Although not specific to reflexology, we have found an increase in baroreceptor reflex sensitivity while Frankel ${ }^{35}$ found decreases in this parameter after reflexology. This contradictory result may be due to the different techniques used to assess baroreflex function. Our method, the sequence technique, $36,46,50$ is based on the non-invasive analysis of spontaneous covariation of systolic blood pressure and inter-beat interval through a continuous beat-to-beat recording of blood pressure. Frankel 35 used the Valsalva manoeuvre, an older and invasive technique based on respiratory manipulations that produces an indirect baroreceptor reflex sensitivity index. As an indication of the validity of our procedure, in the present study we found correlations of about $r=0.683$ between baroreceptor reflex sensitivity and inter-beat interval and $r=0.843$ between baroreceptor reflex sensitivity and heart rate variability, which are totally congruent with expectations based on physiology. ${ }^{28,37}$ Frankel 35 on the other hand found a negative relation between his baroreceptor reflex sensitivity index and respiratory sinus arrhythmia, which is contrary to current physiological knowledge. ${ }^{28,37}$

Our results concerning heart rate variability are consistent with those of Delaney et al. ${ }^{40}$ who also found increases in heart rate variability after myofascial trigger-point massage. However, our results are contrary to those of Joseph et al. ${ }^{38}$ and Zhen et al. ${ }^{39}$ who found decreases in heart rate variability during reflexology in comparison to a relaxed sitting position. This difference could be explained by the differential experimental procedures being used. Firstly, in these two last studies the massage was performed by a mechanical reflexological device while in our study it was carry out by an expert reflexologist. Secondly, they measured heart rate variability during the actual massage while in our study physiological measurement was carried out just before and after massage. Continuous foot stimulation during reflexology probably produces phasic cardiac responses that could interfere with a reliable measure of heart rate variability. ${ }^{26}$ As regards inter-beat interval, the observed decrease is in accordance with previous studies.7,41

The increase observed in blood pressure after reflexological massage is not consistent with previous results, which usually reported decreases in blood pressure in response to reflexology as well as to other kinds of massage. ${ }^{7,41,42,51-55}$ This difference might be explained by the specific reflex-points being stimulated and the strength and depth of the procedure. In our study the circulatory area was one of the selected points and, as shown by the pain ratings, the massage was performed in depth. It could be argued that the increase in blood pressure provoked by reflexology could be a response to pain and negative feelings during

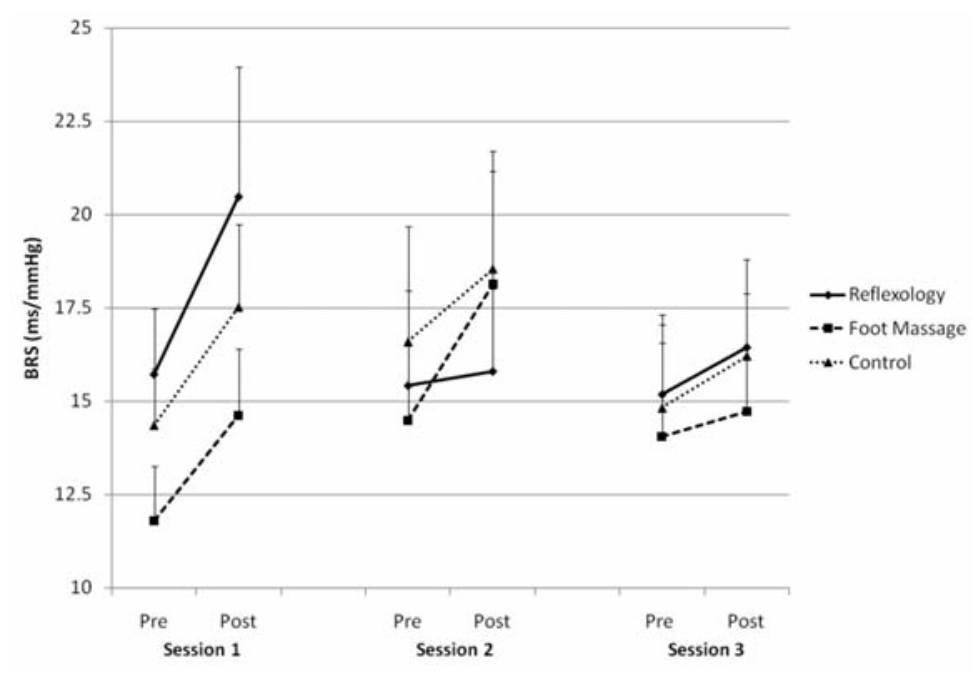

Figure 7. Baroreceptor reflex sensitivity during the three sessions as a function of conditions. Bars indicate standard errors of the mean.

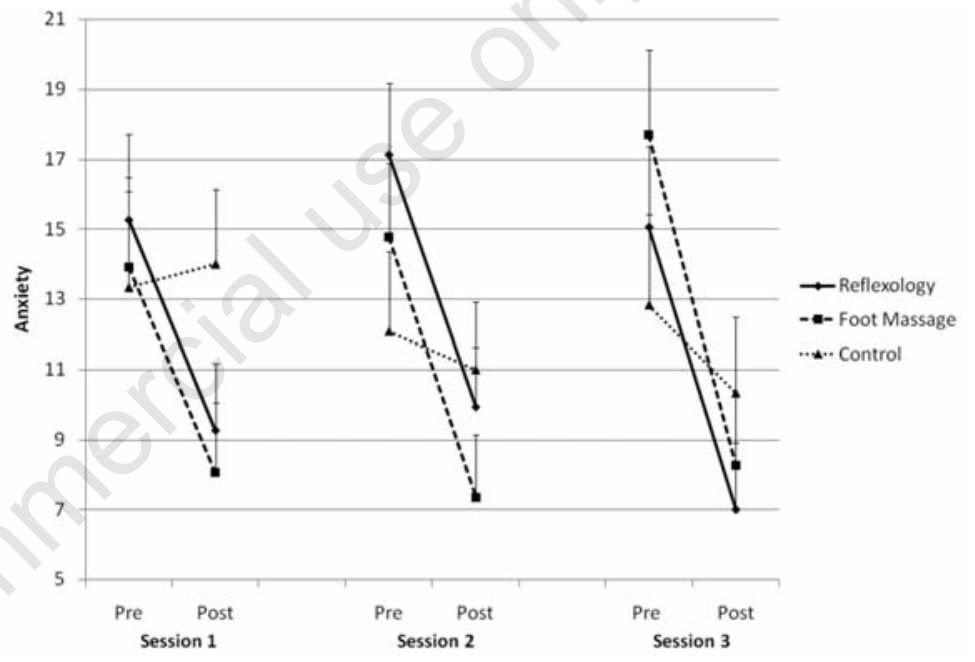

Figure 8. State-anxiety ratings during the three sessions as a function of conditions. Bars indicate standard errors of the mean.

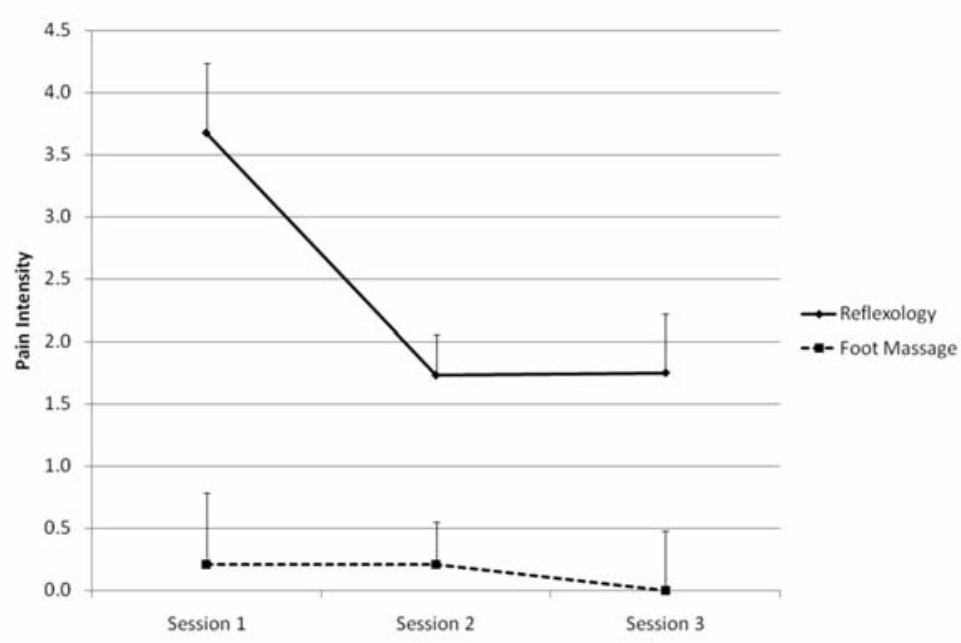

Figure 9. Ratings of pain evoked by the two massage procedures. Bars indicate standard errors of the mean. 
the intervention. Pain is associated with increases in blood pressure 56 and it might explain at least in part the increase in blood pressure after reflexology. However, pain is also accompanied by decreases in inter-beat interval, heart rate variability and baroreceptor reflex sensitivity, 56 which contrast with the actual changes observed in these parameters. Furthermore, participants in the reflexology group also rated the massage as pleasant and their state anxiety decreased after the intervention. In fact, no differences were found between reflexology and foot massage groups in terms of anxiety and pleasantness ratings. Altogether the observed increases in inter-beat interval, heart rate variability and baroreceptor reflex sensitivity and the self-reported data suggest that the pain hypothesis can be discarded in our study.

An underlying physiological mechanism in the explanation of the therapeutic effects of reflexology and massage therapies in general may be that they evoke a relaxation response. ${ }^{13}$ Relaxation is known to have several beneficial effects such as an increase in parasympathetic activity, a decrease in sympathetic activity, catecholamine and cortisol levels, immunologic potentiation, etc. ${ }^{57}$ All these effects promote the normalization/balancing of any deficiency and allow the body to function more efficiently. ${ }^{57}$ The decrease observed in state anxiety along with the changes in interbeat interval, heart rate variability and baroreceptor reflex sensitivity (that suggests an increase in parasympathetic tone), which we observed in the reflexology and foot massage groups are congruent with this hypothesis. However, the associated increase in blood pressure due to reflexology is contrary to a relaxation response and suggests that the therapeutic effect of reflexology is different and cannot simply be assimilated to the relaxation response. Therefore, reflexology seems to act through a different mechanism.

As previously stated, the increase in heart rate variability and baroreceptor reflex sensitivity suggest an increase in cardiac vagal activity after our three experimental conditions. The increase in blood pressure associated specifically with the reflexology therapy suggests a simultaneous activation of sympathetic cardiac control. In this way, one of the main findings of the study is the occurrence of a co-activation of both branches of the Autonomic Nervous System (ANS) after reflexology. Co-activation of the two branches of the ANS is one of the multiple possible modes of autonomic functioning within a 2-dimensional (sympathetic / parasympathetic) space. ${ }^{58}$ 0ne might speculate that reflexology strengthens the balance of the ANS and makes it work in equilibrium with superior level of activity of both branches. This overall higher activity of the ANS would help to optimize homeostatic activity and increase the organism's capacity to respond adaptatively to internal and external challenges.

Reflexology is believed to stimulate a healing process, probably by potentiating a homeostatic mechanism that restores health status. Blood pressure is associated with the delivery of nutrients, oxygen and energy to the tissues as well as the removal of damaging toxins from the body. One could speculate that the start of a healing process, as with any other adaptive processes, needs some additional energy resources. In this way the increase in blood pressure is a useful mechanism to mobilize and distribute this need for supplemental energy.

Reflexologists claim that the healing effects that this technique may generate are accumulative. Our findings show that the increases produced by reflexology on blood pressure increases the more sessions are performed. Most previous studies tested the efficacy of reflexology after just one session. Our results confirm the idea of an accumulative effect of reflexology 59 and suggest the necessity of including several sessions in future investigations.

Finally, an unexpected and surprising result from our study is the increase in inter-beat interval, heart rate variability and baroreceptor reflex sensitivity found in the waiting-time control group. This clearly shows that the habituation process associated simply with the course of time is able to produce significant changes in cardiovascular variables that resemble some of the changes associated with massage. This result is of relevance and suggests the need for the addition of waiting-time control groups in future studies on the effects of alternative therapies on physiological indices. Similarly, the observed decrease in blood pressure over the sessions regardless of the condition could also reflect a more long-term habituation process to the laboratory situation.

To conclude, the results of this study show that reflexology, foot-massage and even simply the passing of time increase inter-beat interval, heart rate variability and baroreceptor reflex sensitivity, resulting in relaxation-like changes. However, reflexology specifically is associated with an increase in blood pressure not observed in the other conditions. These results suggest that reflexology is associated with a co-activation of the two branches of the ANS, which, combined with the increased supply of energy associated with the rise in blood pressure, may help optimize homeostatic activity and promote the healing process.

\section{References}

1. Blunt E. Foot Reflexology. Holist Nurs Pract 2006;20:257-9.

2. Oleson T, Flocco W. Randomized controlled study of premenstrual symptoms treated with ear, hand, and foot reflexology. Obstet Gynecol 1993;82:906-11.

3. Quinn F, Hughes CM, Baxter GD. Refle-xology in the management of low back pain: a pilot randomised controlled trial. Complement Ther Med 2008;16: 3-8.

4. Wang XM. Treating type II diabetes mellitus with foot reflexotherapy. Chung-Kuo Chung Hsi i His Chieh Ho Tsa Chih 1993;13:536-8.

5. Stephenson NL, Weinrich SP, Tavakoli AS. The Effects of foot reflexology on anxiety and pain in patients with breast and lung cancer. Oncol Nurs Forum 2000;27:67-72.

6. Siev-Ner I, Gamus D, Lerner-Geva L, Achiron A. Reflexology treatment relieves symptoms of multiple sclerosis: a randomized controlled study. Mult Scler 2003;9: 356-61.

7. McVicar AJ, Greenwood CR, Fewell F, et al. Evaluation of anxiety, salivary cortisol and melatonin secretion following reflexology treatment: a pilot study in healthy individuals. Complement Ther Clin Pract 2007; 13:137-45.

8. Petersen LN, Faurschou R, Olsen OT, Svendsen UG. Foot zone therapy and bronchial asthma: a controlled clinical trial. Ugeskr Laeger 1992;154:2065-8.

9. Ernst E. Is reflexology an effective intervention? A systematic review of randomised controlled trials. Med J Aust 2009; 191:263-6.

10. Nakamaru T, Miura N, Fukushima A, Kawashima R. Somatotopical relationships between cortical activity and reflex areas in reflexology: A functional magnetic resonance imaging study. Neurosci Lett 2008;448:6-9.

11. Botting D. Review of literature on the effectiveness of reflexology. Complement Ther Nurs Midwifery 1997;3:123-30.

12. Byers DC. Better health with foot reflexology. Saint Petersgurg, FL: Inghan; 1994.

13. Moyer CA, Rounds J, Hannum JW. A metaanalysis of massage therapy research. Psychol Bull 2004;130:3-18.

14. Grewen KM, Girdler SS, Amico J, Light KC. Effects of partner support on resting oxytocin, cortisol, norepinephrine, and blood pressure before and after warm partner contact. Psychosom Med 2005;67:531-8.

15. Light KC, Grewen KM, Amico J. More frequent partner hugs and higher oxytocin levels are linked to lower blood pressure and heart rate in premenopausal women. Biol Psychol 2005;69:5-21.

16. Knox SS, Uvnäs-Moberg K. Social isolation and cardiovascular disease: an atherosclerotic pathway? Psychoneuroendocrinology 1998;23:877-90.

17. Tailor SE, Klein LC, Lewis BP, et al. Biobehavioral responses to stress in females: tend-and-befriend, not fight-or-flight. Psy- 
chol Rev 2000;107:411-29.

18. Uvnas-Moberg K. The oxytocin Factor. Boston: Perseus Press; 2004.

19. Gallace A, Spence C. The science of interpersonal touch: an overview. Neurosci Biobehav Rev 2010;34:246-59.

20. Thayer J, Lane R. A model of neuro-visceral integration in emotion regulation and dysregulation. J Affect Disord 2000;61:20116.

21. Thayer J, Brosschot J. Psychosomatics and psychopathology: looking up and down from the brain. Psychoneuroendocrinology 2005;30:1050-8.

22. Thayer JF, Lane RD. The role of vagal function in the risk for cardiovascular disease and mortality. Biol Psychol 2007;74:224-42.

23. Sleight $P$. The importance of the autonomic nervous system in health and disease. Aust N Z J Med 1997;27:467-73.

24. La Rovere MT, Bigger TJ, Marcus FI, et al. Baroreflex sensitivity and heart rate variability in prediction of total cardiac mortality after myocardial infarction. Lancet 1998;351:478-84.

25. Reyes del Paso GA, Garrido S, Pulgar A, et al. Aberrances in autonomic cardiovascular regulation in fibromyalgia síndrome and their relevance for clinical pain reports. Psychosom Med 2010;72:462-70.

26. Task Force of the European Society of Cardiology and the North American Society of Pacing and Electrophysiology. Heart rate variability: standards of measurement, physiological interpretation and clinical use. Circulation 1996;93:1043-65.

27. Eckberg D, Sleight P. Human baroreflexes in health and disease. Oxford: Oxford University Press; 1992.

28. Reyes del Paso GA, Langewitz W, Robles H, Perez N. A between-subjects comparison of respiratory sinus arrythmia and baroreceptor cardiac reflex sensitivity as noninvasive measures of tonic parasympathetic cardiac control. Int J Psychophysiol 1996;22:163-71.

29. Sleight P, La Rovere MT, Mortara A, et al. Physiology and pathophysiology of heart rate and blood pressure variability in humans: is power spectral analysis largely an index of baroreceptor gain?. Clin Sci 1995; 88:103-9.

30. Rau H, Elbert T. Psychophysiology of arterial baroreceptors and the etiology of hypertension. Biol Psychol 2001;57:179201.

31. Duschek S, Mück I, Reyes del Paso GA. Relationships between baroreceptor cardiac reflex sensitivity and pain experience in normotensive individuals. Int $\mathrm{J}$ Psychophysiol 2007;65:193-200.

32. Reyes del Paso GA, González MI,
Hernández JA, et al. Tonic blood pressure modulates the relationships between baroreceptor cardiac reflex sensitivity and cognitive performance. Psychophysiology 2009;46:932-8.

33. Lehrer PM, Vaschillo E, Vaschillo B. Resonant frequency training to heart cardiac variability: Rationale and manual for training. Appl Psychophysiol Biofeedback 2000;25:177-91.

34. Lehrer PM, Vaschillo E, Vaschillo B, et al. Heart rate variability biofeedback increases baroreflex gain and peak expiratory flow. Psychosom Med 2003;65:796-805.

35. Frankel BSM. The effect of reflexology on baroreceptor reflex sensitivity, blood pressure and sinus arrhythmia. Complement Ther Med 1997;5:80-4.

36. Parati G, di Rienzo M, Mancia G. How to measure baroreflex sensitivity: From the cardiovascular laboratory to daily life. J Hypertens 2000;18:7-19.

37. Reyes del Paso GA, Hernández JA, González MI. Differential analysis in the time domain of the baroreceptor cardiac reflex sensitivity as a function of sequence length. Psychophysiology 2004;41:483-88.

38. Joseph KP, Acharya RU, Poo CK, et al. Effect of reflexological stimulation on heart rate variability. ITBM-RBM 2004;25: 40-5.

39. Zhen LP, Fatimah SN, Acharya RU, et al. Study of heart rate variability due to reflexological stimulation. Clin Acupunct Oriental Med 2004;4:173-8.

40. Delaney JPA, Leong KS, Watkins A, Brodie D. The short-term effects of myofascial trigger point massage therapy on cardiac autonomic tone in healthy subjects. J Adv Nurs 2002;37:364-71.

41. Kaye AD, Kaye AJ, Swinford J, et al. The effect of deep-tissue massage therapy on blood pressure and heart rate. J Altern Complement Med 2008;14:125-8.

42. Ejindu A. The effects of foot and facial massage on sleep induction, blood pressure, pulse and respiratory rate: Crossover pilot study. Complement Ther Clin Pract 2007;13:266-75.

43. Tochikubo 0, Ri S, Kura N. Effects of Pulse-Synchronized Massage With Air Cuffs on Peripheral Blood Flow and Autonomic Nervous System. Circ J 2006;70: 1159-1163.

44. Settels JJ, Wasseling KH. FIN.A-PRES: Non-invasive finger arterial pressure waveform registration. In: Orlebeke JF, Mulder G, vaan Doornen LPJ, editors. The psychophysiology of cardiovascular control. New York: Plenum Presp; 1985. pp 267-283.

45. Spielberger CD, Gorsuch RL, Lushene R.
Manual del Cuestionario de Ansiedad Estado/Rasgo (STAI) $3^{\text {a }}$ ed. Madrid: TEA; 1982.

46. Reyes del Paso GA, González MI, Hernández JA. Comparison of baroreceptor cardiac reflex sensitivity estimates from inter-systolic and ECG R-R intervals. Psychophysiology, 2010;47:1102-8.

47. Steptoe A, Vögele C. Cardiac baroreceptor reflex function during postural change assessed using non-invasive spontaneous sequence analysis in young men. Cardiovas Res 1990;24:627-32.

48. Berntson GG, Lozano DL, Chen YJ. Filter properties of root mean square successive difference (RMSSD) for heart rate. Psychophysiology 2005;42:246-52.

49. Reyes del Paso GA. An on-line program to calculate respiratory sinus arrhythmia amplitude. Behav Res Med Inst Comp 1992;24:464-6.

50. Reyes del Paso GA, González MI, Hernández JA. Comparison of baroreceptor cardiac reflex sensitivity estimates from inter-systolic and ECG R-spike intervals. Psychophysiology 2010;47:1102-8.

51. Mackereth PA, Booth K, Hillier VF, Caress AL. Reflexology and progressive muscle relaxation training for people with multiple sclerosis: A crossover trial. Complement Ther Clin Pract 2009;15:14-21.

52. Post-White J, Kinney ME, Savik K, et al. Therapeutic massage and healing touch improve symptoms in cancer. Integr Cancer Ther 2003;2:332-44.

53. Won JS, Kim KS, Kim KH, et al. Effect of foot massage on stress in student nurses in clinical practice. J Korean Acad Nurs 2000;7:197-207.

54. Hernandez-Reif M, Field T, Krasnegor J, et al. High blood pressure and associated symptoms were reduced by massage therapy. J Bodyw Mov Ther 2000;4:31-8.

55. Field TM. Massage therapy effects. Am Psychol 1998;53:1270-81.

56. Duschek S, Reyes del Paso, GA. Quantification of cardiac baroreflex function at rest and during autonomic stimulation. J Physiol Sci 2007;57:259-68.

57. Berson H, Klipper MZ. The relaxation response. New York: HarperTorch; 2000.

58. Berntson GG, Cacioppo JT, Quigley KS. Autonomic determinism: The modes of autonomic control, the doctrine of autonomic space, and the laws of autonomic constraint. Psychol Rev 1991;98:459-87.

59. Norman L. The reflexology handbook. London: Piatkus; 1992. 